EDITORIAL

\title{
Health research towards nation development
}

The term health research, also called medical research, refers to research that is conducted to learn more about human health. Health research aims at finding better ways to prevent and treat diseases and paves the way to improve the care and wellbeing of people worldwide. Further, it is meant to positively contribute to the process of ensuring health equity across the world and the overall development of nations. Many developing countries, including Sri Lanka, have made substantial investments in building and enhancing the capacities for research in health and related fields. Such efforts by the low and middle income countries have been supplemented through international programmes sponsored by developed nations that promote education, training through scholarships and fellowships, networking and collaborative programmes with resultant capacity building of local professionals in all spheres including health and related fields.

Despite decades of such efforts, the overall picture of progress is a mixed one and what is apparent in Sri Lanka is no exception. Too often, the investments have failed to result in significant impacts on health policies and practices. A mutually reinforcing set of factors, including brain-drain, lack of sustained investment in research capacity, lack of alignment of funders' goals and researchers' interests with national priorities, and failure by policy makers and practitioners to use research to inform their decisions, could be considered as cyclical causes and effects. As a result, members of the research community frequently complain that they are undervalued, their potential contribution ignored and that there is an absence of a culture of utilization of research to inform policy; while their work is often regarded as remote and irrelevant by potential users of evidence and innovation.

Since there is general acceptance that research should play a critical role in strengthening health systems, improving the equitable distribution of high quality services and advancing human development, there is an obvious need for a strong, transparent and sustainable national health research system, whose primary purpose should be to generate relevant knowledge. Building and sustaining research capacity is an essential and effective means of accelerating research contributions to health and development. Nurturing individual scientific competence and leadership, strengthening institutions, establishing strong linkages between research and action agencies, and reinforcing national institutions through international networks are all important elements of capacity building (CHRD, 1990).

The United Nations Development Programme (UNDP) defines research capacity strengthening (RCS) as 'the process by which individuals, organizations and societies develop abilities (individually and collectively) to perform functions effectively, efficiently and in a sustainable manner to define problems, set objectives and priorities, build sustainable institutions and bring solutions to key national problems' (UNDP, 1998). This definition makes it clear that RCS refers to a broad and complex area, dealing not only with skills and competencies at various levels and by various actors, but also with expected outcomes and impacts on policies and programmes. Therefore, a comprehensive framework, which covers the various dimensions, functions and actors in RCS could be helpful to develop a proper understanding of RCS conceptually and to address its major challenges effectively in action terms.

For health research of a country to have an impact on health status of its people and contribute to social and economic development and innovation, it is generally accepted that RCS must involve a 'systems approach'. This is for balancing long term investments at three levels: firstly, the individual investigator (training and research support), secondly, the institutions and organizations in which they work and thirdly, the national and regional health research systems that can provide a supportive environment for sustainable growth and improvement of a country's health research capacity (Essence, 2011).

The twenty first century has presented us with many new challenges related to health and wellbeing of people. Issues related to rapidly ageing populations, urbanization, 
climate change, food security and water availability are just a few examples. Health issues such as obesity, diabetes, hypertension, threats due to newly emerged or re-emerged pathogens and mental health disorders are becoming increasingly important from a public health perspective. The fact that these challenges are common to most countries across the globe enables more concerted national and global action to make RCS a priority in all countries and assure true partnerships that allow local scientists to be involved at each and every level of the process to address these shared global health problems. It is important to create a conducive environment that would enable the effective use of modern tools and technology in fields such as genomics, proteomics, molecular epidemiology and diagnostics using chip technology etc. that are advancing at a tremendously fast pace to tackle modern-day health issues. Furthermore, if we are to reap the full benefits of scientific advancements, efforts should be made through scientific research and innovation to create and/or modify tools that are better suited to the country's needs and social context. Sri Lanka is not unique in facing the challenge of translating what is known into interventions that improve health via reaching the populations that need these, most in a timely and cost-effective manner (Eliss \& Glass, 2011). Therefore, It is important that proper assessment is made to gauge the adequacy of current research capacity and to identify the gaps that need to be addressed in order to fulfill the health and development needs of the country. This should go hand in hand with adequate investments in RCS, including education and training of young scientists and to create an enabling environment for research with the establishment and maintenance of strong educational and research institutions that produce and host researchers in order to minimize braindrain. Collaborative programmes through international agencies should also be encouraged to adopt a systems approach towards RCS in their assistance programmes. Finally, national health research policies should support the above measures in order to ensure that the outputs of health research would strengthen the health system of the country, improve the equitable distribution of high quality services and would meaningfully contribute to the advancement of the country's development.

\section{REFERENCES}

1. Commission on Health Research for Development (CHRD) (1990). Health Research: Essential Link to Equity in Development. Oxford University Press, New York, USA.

2. Eliss R. \& Glass R. (2011). Gaps in research. Global Health Magazine 9: 6 - 8 .

3. Essence (2011). Planning, monitoring and evaluation. Framework for capacity strengthening in health research. Essence Good Practice Document Series. Available at http:// apps.who.int/tdr/svc/publications/non-tdr-publications/ essence-framework.

4. United Nations Development Programme (UNDP) (1998). Capacity assessment and development in a systems and strategic management context. Technical Advisory Paper, No. 3. Bureau for Development Policy, UNDP, New York, USA.

Nadira D. Karunaweera 